\title{
Crossover behaviors in branching annihilating attracting walk
}

\author{
Su-Chan Park (박수찬) \\ The Catholic University of Korea, Bucheon 14662, Republic of Korea
}

(Dated: May 8, 2020)

\begin{abstract}
We introduce branching annihilating attracting walk (BAAW) in one dimension. The attracting walk is implemented by a biased hopping in such a way that a particle prefers hopping to a nearest neighbor located on the side where the nearest particle is found within the range of attraction. We study the BAAW with four offspring by extensive Monte Carlo simulation. At first, we find the critical exponents of the BAAW with infinite range of attraction, which are different from those of the directed Ising (DI) universality class. Our results are consistent with the recent observation [B. Daga and P. Ray, Phys. Rev. E 99, 032104 (2019)]. Then, by studying crossover behaviors, we show that as far as the range of attraction is finite the BAAW belongs to the DI class. We conclude that the origin of non-DI critical behavior of the BAAW with infinite range of attraction is the long-range nature of the attraction.
\end{abstract}

\section{INTRODUCTION}

Various one dimensional models with two (sets of) symmetric absorbing states, which are also revealed as modulo- 2 conservation in dynamics of domain walls between two different absorbing states, and with finite range of interaction have been found to belong to the directed Ising (DI) universality class [1 -7]. An infinite barrier between symmetric absorbing states [8] was identified as an important condition for a model to belong to the DI universality class. The validity of this assertion can be checked by studying what will happen if the condition is not met. Indeed, there are (at least) three different ways of breaking the condition and each way mediates distinct crossover from the DI class to the directed percolation (DP) universality class [5, 9, 10]. The short-range interaction is also believed to be an important premise because the long-range Lévy flight changes the critical behavior 11].

Recently, a variant of the branching annihilating random walk (BAW) [2] was introduced in Ref. [12]. In this model, particles hop in a biased manner and the direction of bias changes from configuration to configuration in such a way that hopping toward the nearest particle is preferred. Daga and Ray [12] assumed that the bias is a local interaction because the BAW with odd number of offspring is still found to belong to the DP class even in presence of the bias. This assumption sounds plausible in that a long-range interaction should change the critical behavior of models in the DP class [13 16].

However, the BAW with even number of offspring, which is a typical model in the DI class, does not belong to the DI class any more if the bias is present [12]. If the bias is indeed a local interaction, then this study poses a challenge to understanding the DI universality class. Two symmetric absorbing states with infinite barrier would not be sufficient to determine the DI class.

The purpose of this paper is to investigate whether the bias introduced in Ref. [12] to the BAW with even number of offspring has nothing to do with the long-range interaction. To this end, we come up with a modified version of Ref. 12] by introducing the range of attraction.
We will present how the range of interaction affects the critical behavior.

The structure of the paper is as follows. In Sec. II, we introduce the branching annihilating attracting walk (BAAW) with finite range of attraction. In this model, hopping is biased only if a nearest particle is located within the range of attraction. When the range of attraction is infinite, the BAAW is identical to the model in Ref. [12]. In Sec. III, we present our simulation results in two subsections. In Sec. III A we find the critical exponents of the BAAW with infinite range of attraction (to be abbreviated as $\mathrm{BAAW}_{\infty}$ ) more accurately than Ref. [12] by taking corrections to scaling into account. In Sec. IIIB, we present simulation results for the BAAW with finite range of attraction, focusing on crossover behaviors. Section IV] summarizes our work with discussion.

\section{MODEL}

The BAAW is defined on a one dimensional lattice of size $L$. We always assume periodic boundary conditions. A configuration $C$ at time $t$ is specified by the occupation number $a_{i}$ at each site $i(0 \leq i \leq L-1)$. $a_{i}$ can be either 1 or 0 and multiple occupancy is not allowed. Each particle can either hop to one of its nearest neighbor sites or branch $m$ particles. When a particle hops, the direction is biased toward the closest particle if the closest particle is located within distance $R$. If the distance to the closest particle is larger than $R$, then the hopping is symmetric. Whenever two particles happen to occupy a same site, these two particles undergo a pair-annihilation reaction and are removed from the system immediately.

Now we define the model more explicitly. For ease of presentation, we introduce an indicator $I_{i}(x ; C)$ for a given configuration $C$ as

$$
I_{i}(x ; C) \equiv \delta\left(a_{i+x}, 1\right) \prod_{k=1}^{|x|-1} \delta\left(a_{i+k}, 0\right) \delta\left(a_{i-k}, 0\right),
$$

where $\delta(a, b)$ is the Kronecker delta symbol that is one 
(zero) if $a=b(a \neq b)$. In words, $I_{i}(x ; C)$ is one if in configuration $C$ the nearest occupied site from site $i$ is $i+x$ irrespective of whether site $i-x$ is occupied or not; and zero otherwise. We introduce another indicator functions $\chi_{i}^{ \pm}$,

$$
\chi_{i}^{+} \equiv \sum_{x=1}^{R} I_{i}(x ; C), \quad \chi_{i}^{-} \equiv \sum_{x=1}^{R} I_{i}(-x ; C) .
$$

Note that $\chi_{i}^{+}\left(\chi_{i}^{-}\right)$is 1 if in configuration $C$ the nearest occupied site from $i$ is within distance $R$ and is on the right (left) hand side of $i$; and 0 otherwise. We will refer to $R$ as the range of attraction. Notice that both $\chi^{ \pm}$can be 1 simultaneously if there is a positive $x \leq R$ such that $I_{i}(x ; C)=I_{i}(-x ; C)=1$.

Now we are ready to explain the dynamic rules of the BAAW. Each particle can hop to one of its nearest neighbors with rate $p$ (hopping event) or branch $m$ offspring with rate $1-p$ (branching event). If a particle at site $i$, say, is decided to move in the hopping event, then it hops to $i \pm 1$ with probability

$$
H^{ \pm} \equiv \frac{1}{2} \pm \epsilon\left(\chi_{i}^{+}-\chi_{i}^{-}\right)
$$

where $0 \leq \epsilon \leq \frac{1}{2}$. A particle prefers hopping toward the closest particle if the closest particle is found within $R$.

In the branching event, all sites $j$ with $0<|j-i| \leq$ $\lfloor m / 2\rfloor$ will be given an offspring, where $\lfloor x\rfloor$ is the floor function (greatest integer not larger than $x$ ). If $m$ is odd, then one more particle will be placed at site either $i+(m+1) / 2$ or $i-(m+1) / 2$, which is chosen at random with equal probability. If two particles happen to occupy the same site by dynamics (either hopping or branching), then they are annihilated together (typically represented by $A+A \rightarrow 0$ ) in no time.

Notice that if either $\epsilon=0$ or $R=0$, then the model is the same as the branching annihilating random walk [2], which belongs to the DI class if $m$ is even. Also note that if $\epsilon>0$ and $R=\infty$, then the model is identical to that in Ref. [12]. We fix $\epsilon=0.1$ in the next section, but our preliminary simulations showed that other nonzero value of $\epsilon$ does not alter the conclusion.

We use two different initial conditions. The first initial condition is such that all sites (for finite $L$ ) are occupied. The second one is such that only two consecutive sites are occupied in an infinite lattice $(L=\infty)$. In this case, the site index runs over all integers and the initial configuration can be represented as $a_{0}=a_{1}=1$ and $a_{i}=0$ for $i \neq 0,1$. We will refer to a Monte Carlo simulation with the first (second) initial condition as the steadystate (dynamic) simulation.

In the steady-state simulation, we are interested in the density of occupied sites at time $t$, which is defined as

$$
\rho(t)=\frac{\left\langle M_{t}\right\rangle}{L},
$$

where $M_{t}=\sum_{i} a_{i}$ is the number of occupied sites at time $t$ and $\langle\ldots\rangle$ represents the average over all ensemble.
In actual simulation, $L$ is large enough that a finite-size effect is negligible.

In the dynamic simulation, we measure the mean number of particles $N(t)$ averaged over all ensemble, the (survival) probability $S(t)$ that there is at least one particle in the system at time $t$, and the spreading $R^{2}(t)$ averaged over surviving ensemble. These quantities are formally defined as

$$
\begin{aligned}
& N(t) \equiv\left\langle M_{t}\right\rangle, \\
& S(t) \equiv\left\langle\left(1-\delta_{M_{t}, 0}\right)\right\rangle, \\
& R^{2}(t) \equiv\left\langle\left(k_{M}-k_{m}\right)^{2}\right\rangle / S(t), \\
& k_{M} \equiv\left(1-\delta_{M_{t}, 0}\right) \max \left\{i \mid a_{i}=1\right\}, \\
& k_{m} \equiv\left(1-\delta_{M_{t}, 0}\right) \min \left\{i \mid a_{i}=1\right\}
\end{aligned}
$$

where $\delta_{a, b}$ is the Kronecker $\delta$ symbol.

In all simulations, we measure quantities in question at a regularly spaced time points on a logarithmic scale. To be specific, the $i$ th measurement is performed at $T_{i}$ defined as

$$
T_{i}= \begin{cases}i, & 1 \leq i \leq 40 \\ \left\lfloor 40 \times 2^{(i-40) / 15}\right\rfloor, & 41 \leq i \leq 55 \\ 2 T_{i-15}, & 56 \leq i\end{cases}
$$

Notice that 50 measurement points roughly correspond to a decade on a logarithmic scale.

\section{SIMULATION RESULTS}

\section{A. Infinite $R$}

We begin with presenting simulation results of the BAAW $_{\infty}$ with $m=4$ (four offspring). The BAAW $\infty$ was already studied in Ref. [12], but we will provide more accurate critical exponents and critical points.

We first present the results of the dynamic simulation. At the critical point, the quantities in question exhibit power-law behaviors such as

$$
N(t) \sim t^{\eta}, \quad S(t) \sim t^{-\delta^{\prime}}, \quad R^{2}(t) \sim t^{2 / z}
$$

where $\eta, \delta^{\prime}$, and $z$ are critical exponents. The critical exponents as well as the critical point are estimated by analyzing effective exponents defined as

$$
\begin{aligned}
\eta_{\mathrm{e}}(t ; b) & \equiv \frac{\ln [N(t) / N(t / b)]}{\ln b}, \\
-\delta_{\mathrm{e}}^{\prime}(t ; b) & \equiv \frac{\ln [S(t) / S(t / b)]}{\ln b}, \\
z_{\mathrm{e}}(t ; b) & \equiv \frac{2 \ln b}{\ln \left[R^{2}(t) / R^{2}(t / b)\right]},
\end{aligned}
$$

where $b>1$ is a constant. If the system is at the critical point, then the effective exponents should approach the corresponding critical exponents as $t \rightarrow \infty$. 


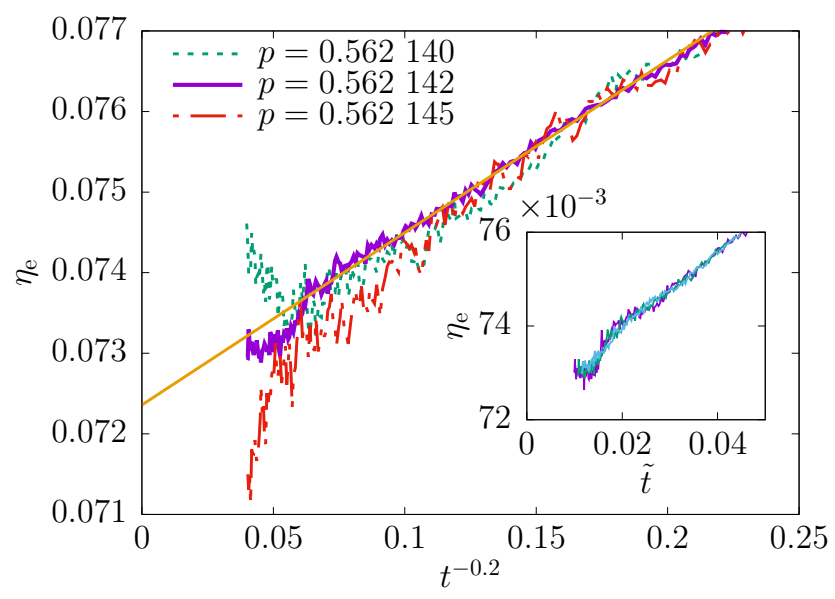

FIG. 1. Plots of $\eta_{\mathrm{e}}$ vs. $t^{-0.2}$ for the $\mathrm{BAAW}_{\infty}$ with $p=$ 0.56214 (dotted green line), 0.562142 (solid purple line), and 0.562145 (dot-dashed red line), top to bottom. Here $b$ is set 16 . The line shows the fitting of the data for $p=$ 0.562142 with a linear function, which intersects the ordinate at 0.0724 . Inset: Plots of $\eta_{\mathrm{e}}$ vs. $\tilde{t} \equiv t^{-0.2}\left(b^{0.2}-1\right) / \ln b$ for $p=0.562142$ with $b=8,16$, and 32. Small $\tilde{t}$ (or large $t$ ) behavior for different $b$ is hardly discernible, indicating that the corrections-to-scaling exponent $\chi_{\eta}$ is estimated properly.

To find the critical exponents accurately, we have to take corrections to scaling into account. If the long time behavior of, for example, $N(t)$ at the critical point is

$$
N(t)=A t^{\eta}\left[1+B t^{-\chi_{\eta}}+o\left(t^{-\chi_{\eta}}\right)\right],
$$

where $o(x)$ stands for all terms that approach zero faster than $x$ for small $x$, then the effective exponent behaves asymptotically as

$$
\eta_{\mathrm{e}}(t ; b) \approx \eta-B \frac{b^{\chi_{\eta}}-1}{\ln b} t^{-\chi_{\eta}} .
$$

At the critical piont, $\eta_{\mathrm{e}}$ shows a linear behavior for large $t$ when it is drawn as a function of $t^{-\chi_{\eta}}$. If the system is in the active (absorbing) phase, then $\eta_{\mathrm{e}}(t)$ should eventually veer up (down) as $t$ increases. Hence, analyzing $\eta_{\mathrm{e}}$ as a function of $t^{-\chi_{\eta}}$ with correct value of $\chi_{\eta}$ is important to find the critical point. The critical exponent $\eta$, in turn, can be found by a linear extrapolation of $\eta_{\mathrm{e}}$ at the critical point. Other exponents can be found in a similar manner.

To find $\chi_{\eta}$, we analyze the corrections-to-scaling function $Q_{\eta}$ defined as [17, 18]

$$
Q_{\eta}(t ; b, \chi)=\frac{\ln N(t)+\ln N\left(t / b^{2}\right)-2 \ln N(t / b)}{\left(b^{\chi}-1\right)^{2}},
$$

whose asymptotic behavior at the critical point is

$$
Q_{\eta}\left(t ; b, \chi_{\eta}\right) \approx B t^{-\chi_{\eta}}
$$

By analyzing how $Q_{\eta}$ behaves asymptotically, we can find $\chi_{\eta}$ without prior knowledge of $\eta$. For consistency check

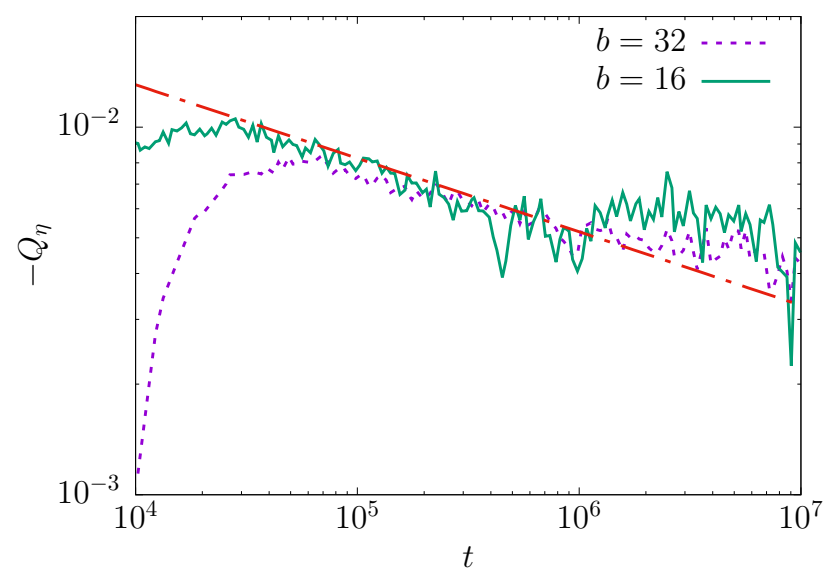

FIG. 2. Double logarithmic plots of $-Q_{\eta}$ vs. $t^{-0.2}$ for $b=16$ and 32 (left to right). The dot-dashed line is $-B t^{-0.2}$ with $B$ $(B<0)$ estimated in Fig. 1

of the estimated $\chi_{\eta}$, we will depict $Q_{\eta}(t ; b, \eta)$ for various $b$ 's, which should behave identically in the asymptotic regime if $\chi=\chi_{\eta}$. In a similar manner, we can also define $Q_{\delta^{\prime}}$ and $Q_{z}$. With our choice of measurement time points in Eq. (6), it is convenient to set $b$ to be of the form $2^{n}$ with positive integer $n$.

Now we present our results. In actual simulations, the maximum observation time is $T_{309} \approx 10^{7}$ and the number of independent runs for $p=0.562142$, which will be shown to be the critical point, is $2.5 \times 10^{8}$.

As a rule of thumb, $\eta$ is the best quantity to find the critical point in the dynamic simulation. So we begin with the analysis of $\eta$. In Fig. 1, we depict $\eta_{\mathrm{e}}$ against $t^{-0.2}$ (that is, we use $\chi_{\eta}=0.2$ ) for different $p$ 's. Since $\eta_{\mathrm{e}}$ for $p=0.56214(0.562145)$ eventually veers up (down) for large $t$ and $\eta_{\mathrm{e}}$ for $p=0.562142$ shows a linear behavior, we conclude that the critical point is $p_{0}=0.562142(3)$, where the number in parentheses indicates the uncertainty of the last digit. Note that our estimate of $p_{0}$ (for $\epsilon=0.1$ ) is more accurate than Ref. [12]. Using a linear extrapolation, we find the critical exponent $\eta=0.0724(5)$. For consistency check, we depict $\eta_{\mathrm{e}}$ vs. $t^{-0.2}\left(b^{0.2}-1\right) / \ln b$ at the critical point for $b=8,16$, and 32 in the inset of Fig. 1. As expected from Eq. (12), these data lie on a single line.

We actually estimated $\chi_{\eta}$ from the analysis of $Q_{\eta}$ at $p=p_{0}$. First note that $B$ in Eq. (12) is negative as $\eta_{\mathrm{e}}$ approaches $\eta$ from above. Therefore, we draw $-Q_{\eta}(t)$ in Fig. 2 for $b=16$ and 32 on a double logarithmic scale. For comparison, we also depict $-B t^{-0.2}$ with $B$ estimated when we found $\eta$ by a linear extrapolation. Consistently with Eq. (14), the asymptotic behavior for different $b$ 's collapses into a single line, which concludes that $\chi_{\eta}$ is indeed 0.2 .

In a similar fashion, we find $\delta^{\prime}$ and $z$ using the corresponding effective exponent. Figure 3 shows the result of our analysis. We have first found that $Q_{\delta^{\prime}} \sim t^{-0.2}$ and $Q_{z} \sim t^{-0.5}$ (details not shown here). Linear extrapola- 

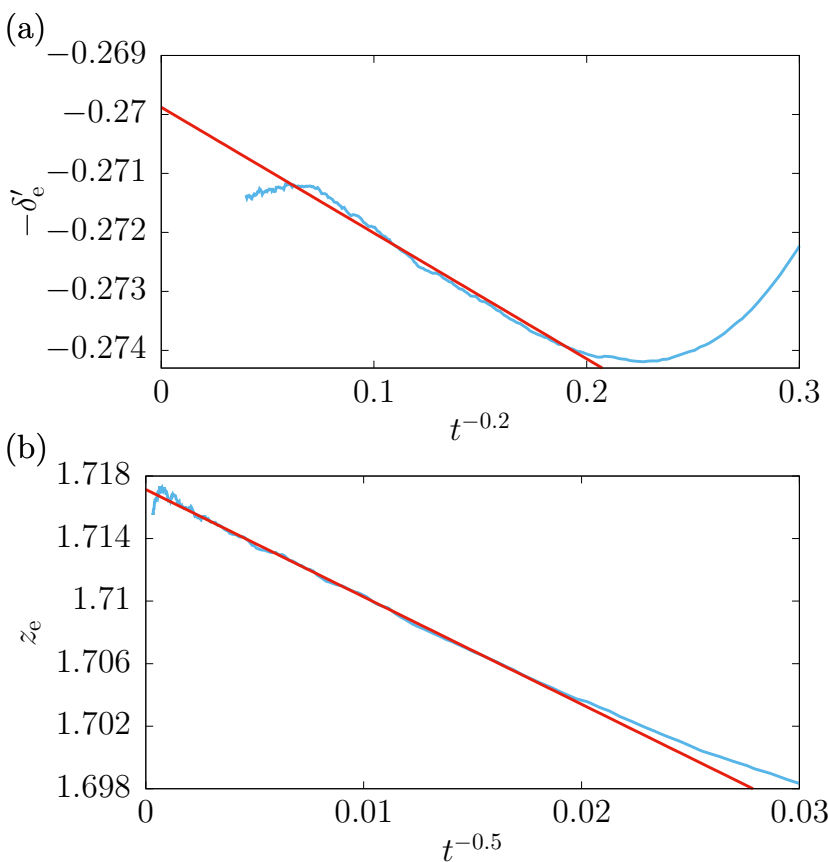

FIG. 3. Plots of the effective exponents at the critical point against $t^{-\chi}$. Here $b$ is set 16 . The lines are results of fitting with a linear function. (a) The effective exponent $-\delta_{\mathrm{e}}^{\prime}$ with $\chi=0.2$. (b) The effective exponent $z_{\mathrm{e}}$ with $\chi=0.5$.

tions give $\delta^{\prime}=0.270(1)$ and $z=1.7170(5)$.

Now we move on to the steady-state simulation. The density $\rho(t)$ is expected to decay at the critical point $p_{0}$ as

$$
\rho(t)=A_{\rho} t^{-\delta}\left[1+B_{\rho} t^{-\chi_{\rho}}+o\left(t^{-\chi_{\rho}}\right)\right],
$$

where $A_{\rho}$ and $B_{\rho}$ are constants. As in the analysis of the dynamic simulation, we study the effective exponent and the corrections-to-scaling function, defined as

$$
\begin{aligned}
-\delta_{\mathrm{e}} & \equiv \frac{\ln [\rho(t) / \rho(t / b)]}{\ln b} \approx-\delta-B_{\rho} \frac{b^{\chi_{\rho}}-1}{\ln b} t^{-\chi_{\rho}}, \\
Q_{\delta} & \equiv \frac{\ln \rho(t)+\ln \rho\left(t / b^{2}\right)-2 \ln \rho(t / b)}{\left(b^{\chi_{\rho}}-1\right)^{2}} \approx B_{\rho} t^{-\chi_{\rho}},
\end{aligned}
$$

where we also presented the asymptotic behaviors at the critical point.

We simulated 1600 independent runs for the system of size $L=2^{22}$ to $t=T_{309} \approx 10^{7}$ at the critical point $p_{0}$. In Fig. 4, we depict $-\delta_{\mathrm{e}}$ against $t^{-0.3}$, where we use $Q_{\delta} \sim t^{-0.3}$; see the inset of Fig. 4. By an extrapolation, we find $\delta=0.2394(3)$. Notice that unlike the DI class $\delta$ is different from $\delta^{\prime}$.

To check whether the scaling relation (see, e.g., Ref. [19])

$$
\delta=\frac{1}{z}-\eta-\delta^{\prime}
$$

is satisfied, we depict the corresponding effective exponents in Fig. 5, which shows an excellent agreement.

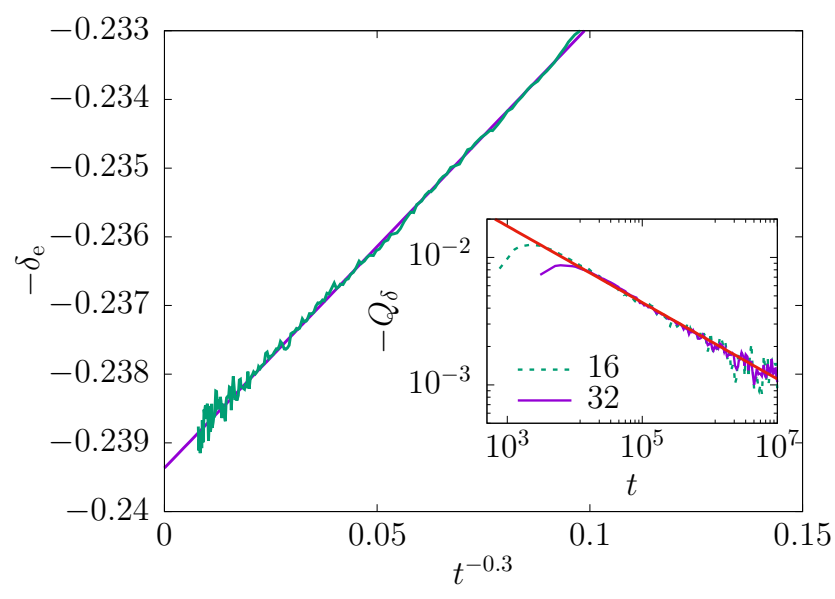

FIG. 4. Plot of $-\delta_{\mathrm{e}}$ vs. $t^{-0.3}$ with $b=16$ at the critical point. The line is the result of the linear fitting. Inset: Plot of $-Q_{\delta}$ vs. $t$ on a double logarithmic scale for $b=16$ and 32 . The slope of the line is -0.3 .

Now we find $\beta$ by the steady-state simulation. To this end, we simulated the system at $p=p_{0}-\Delta$ with $\Delta=$ $\Delta_{n} \equiv 2^{n / 2} \times 10^{-4}$. In actual simulations, $n$ ranges from 7 to $18(n=7,8, \ldots, 18)$. We will denote the steady-state density at $p=p_{0}-\Delta_{n}$ by $\rho_{n}$. To find the steady-state density, we simulated the system of size $L=2^{21}$ and averaged density for $t>2 \Delta_{n}^{-2.53}$ (in fact, 2.53 is the estimated value of the critical exponent $\nu_{\|}$; see below). The number of independent runs ranges from $500(n=$ 18) to $4000(n=7)$.

To the leading order of corrections to scaling, we expect

$$
\rho_{n}=A_{\beta} \Delta_{n}^{\beta}\left[1+B_{\beta} \Delta_{n}^{\chi}+o\left(\Delta_{n}^{\chi}\right)\right],
$$

where $A_{\beta}$ and $B_{\beta}$ are constants. To estimate $\beta$, we also analyzed the effective exponent $\beta_{\mathrm{e}}$ defined as

$$
\beta_{\mathrm{e}}(n ; k)=\frac{\ln \left(\rho_{n+k} / \rho_{n}\right)}{\ln b} \approx \beta+B_{\beta} \frac{b^{\chi}-1}{\ln b} \Delta_{n}^{\chi},
$$

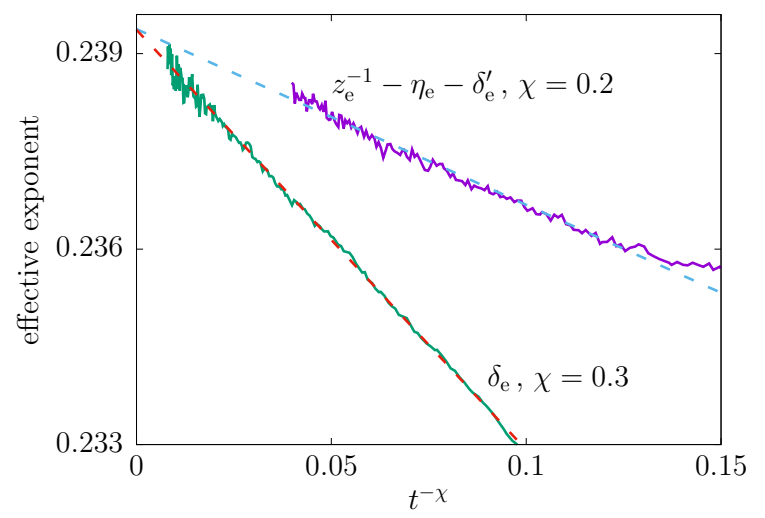

FIG. 5. Plots of effective exponents as a function of $t^{-\chi}$ for $\delta_{\mathrm{e}}$ with $\chi=0.3$ (bottom) and $z_{\mathrm{e}}^{-1}-\eta_{\mathrm{e}}-\delta_{\mathrm{e}}^{\prime}$ with $\chi=0.2$ (top). Here $b$ is 16 . The dashed lines show extrapolations. 


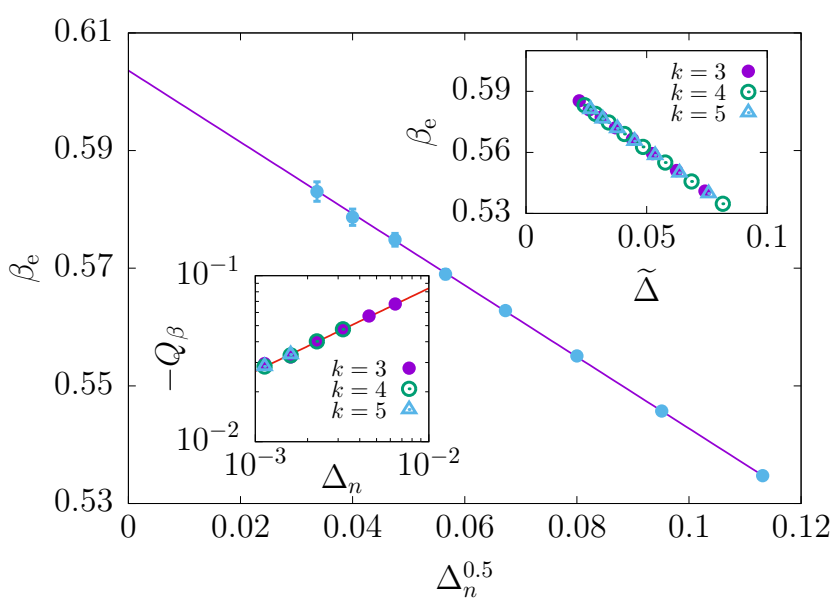

FIG. 6. Plots of $\beta_{\mathrm{e}}$ vs. $\Delta_{n}^{0.5}$ for $k=4\left(b=2^{k / 2}=4\right)$. The line is the result of a linear fitting. Inset: (bottom) Plots of $-Q_{\beta}$ vs. $\Delta_{n}$ for $k=3,4,5$ on a double logarithmic scale. The slope of the line is 0.5 ; (top) plots of $\beta_{\mathrm{e}}$ vs. $\widetilde{\Delta}$ for $b=2 \sqrt{2}, 4$, and $4 \sqrt{2}$, where $\widetilde{\Delta} \equiv \Delta_{n}^{0.5}\left(b^{0.5}-1\right) / \ln b$.

where $b=2^{k / 2}(k=1,2, \ldots)$. To find $\chi$, we analyzed the corrections-to-scaling function [20]

$$
Q_{\beta}(n) \equiv \frac{\ln \rho_{n+2 k}+\ln \rho_{n}-2 \ln \rho_{n+k}}{\left(b^{\chi}-1\right)^{2}} \approx B_{\beta} \Delta_{n}^{\chi}
$$

If the average is taken over $M$ configurations (recall that the measurement time is evenly distributed on a logarithmic scale), then we estimate the statistical error as $e_{n}=3 \times \sigma / \sqrt{M}$, where $\sigma$ is the standard deviation of the measurement. In our calculations, $M$ is generally larger than $10^{4}$.

Using the statistical error $e_{n}$ of the stationary-state density, we also estimate the error of $\beta_{\mathrm{e}}$ by

$$
\beta^{ \pm}=\frac{1}{\ln b} \ln \frac{\rho_{n+k}^{0} \pm e_{n+k}}{\rho_{n}^{0} \mp e_{n}}, \quad \beta^{-}<\beta_{\mathrm{e}}<\beta^{+},
$$

where $\rho_{n}^{0}$ is the average from simulations.

In Fig. 6, we show the behavior of $\beta_{\mathrm{e}}$ obtained using $b=4$ (or $k=4$ ). Data points of $\beta_{\mathrm{e}}$ nicely lie on a line, when it is drawn as a function of $\Delta_{n}^{0.5}$. We actually estimated $\chi$ from the behavior of $Q_{\beta}(n)$, which shows $Q_{\beta} \sim \Delta_{n}^{0.5}$ irrespective of the value of $k$; see the bottom inset of Fig. 6] In the top inset of Fig. 6, we also depict $\beta_{\mathrm{e}}$ against $\widetilde{\Delta} \equiv \Delta_{n}^{0.5}\left(b^{0.5}-1\right) / \ln b$ for various $b$ 's. As anticipated, $\beta_{\mathrm{e}}$ as a function of $\widetilde{\Delta}$ does not exhibit any $b$ dependence for small $\widetilde{\Delta}$, which, in turn, supports that our choice of $\chi$ is legitimate. Finally, by a linear extrapolation, we find $\beta=0.604(2)$.

In Table I] we summarized the critical exponents for the $\mathrm{BAAW}_{\infty}$ with comparison to the DI critical exponents.
TABLE I. Critical exponents of the BAAW $\infty$ and the DI class. The exponents of the DI class are taken from Refs. [17] and [20].

\begin{tabular}{lll}
\hline \hline Exponent & $\mathrm{BAAW}_{\infty}$ & $\mathrm{DI}$ \\
\hline$\eta$ & $0.0724(5)$ & $0.0000(2)$ \\
$\delta^{\prime}$ & $0.270(1)$ & $0.2872(2)$ \\
$z$ & $1.7170(5)$ & $1.7415(5)$ \\
$\delta$ & $0.2394(3)$ & $0.2872(2)$ \\
$\beta$ & $0.604(2)$ & $1.020(5)$ \\
$\nu_{\perp}$ & $1.469(8)$ & $2.04(1)$ \\
$\nu_{\|}$ & $2.53(1)$ & $3.55(2)$ \\
\hline \hline
\end{tabular}

\section{B. Crossover behavior for finite $R$}

Now we move on to the BAAW with finite range of attraction. Before showing the simulation data, let us first ponder on the effect of finite $R$. When $R$ is finite and the system is very close to the critical point, the mean distance between particles can be much larger than $R$. Since the distance between a particle and its nearest particle is mostly larger than $R$, hopping becomes effectively unbiased. Thus, the BAAW with finite $R$ should belong to the DI class.

Since the $\mathrm{BAAW}_{\infty}$ was unequivocally shown not to belong to the DI class, there should be a crossover behavior for sufficiently large but finite $R$. This crossover is described by a scaling function (see, e. g., Refs. [21, 22])

$$
\rho(t ; p, R)=t^{-\delta} \Psi\left[t R^{-\mu_{\|}}, t^{1 / \nu_{\|}}\left(p-p_{0}\right)\right]
$$

where $p_{0}$ is the critical point for the $\mathrm{BAAW}_{\infty}$ we have found in the above; $\delta$ and $\nu_{\|}$are the critical exponents of the $\mathrm{BAAW}_{\infty}$; and $\mu_{\|}=\nu_{\|} / \phi$ with $\phi$ to be the crossover exponent.

At the critical point $p_{c}(R)$ for finite $R$, the scaling function becomes

$$
\rho\left(t ; p_{c}, R\right)=t^{-\delta} \Psi\left[t R^{-\mu_{\|}}, t^{1 / \nu_{\|}}\left(p_{c}-p_{0}\right)\right] .
$$

Since the scaling function at $p=p_{c}(R)$ for given $R$ should have a single scaling parameter, $p_{c}(R)$ should behave as

$$
p_{c}(R)-p_{0} \sim R^{-1 / \phi}
$$

Thus, the crossover exponent can be found by analyzing how $p_{c}$ behaves for large $R$.

To find the critical points, we exploit the fact that a plot of $\rho(t) t^{\delta_{\mathrm{DI}}}$, where $\delta_{\mathrm{DI}}$ is the critical decay exponent of the DI class, should eventually veer up (down) if $p<p_{c}$ $\left(p>p_{c}\right)$, where $\rho(t)$ is the density at time $t$ in the steadystate simulation (details not shown here). The critical points we have found are summarized in Table II.

In Fig. [7 we show that $p_{c}-p_{0}$ is well approximated by a power-law function with $1 / \phi=0.72(2)$, or $\phi=1.39(4)$, which together with $\nu_{\|}$in Table $\llbracket$ gives $\mu_{\|}=1.82(2)$. 
TABLE II. Critical points of the BAAW for various values of the range of attraction $R$. Numbers in parentheses indicate errors in the last digits.

\begin{tabular}{ll}
\hline \hline$R$ & $p_{c}$ \\
\hline 20 & $0.57441(4)$ \\
40 & $0.569475(25)$ \\
80 & $0.56662(3)$ \\
160 & $0.564875(25)$ \\
320 & $0.56379(2)$ \\
$\infty$ & $0.562142(3)$ \\
\hline \hline
\end{tabular}

If we set $p=p_{0}$ for finite $R$ in Eq. (22), then the scaling function becomes

$$
\rho\left(t ; p_{0}, R\right) t^{\delta}=\Psi\left(t R^{-\mu_{\|}}, 0\right) .
$$

For consistency check, we present a scaling collapse in Fig. 8, using exponents obtained above. The system size for Fig. 8 is $L=2^{20}$ and the number of independent runs ranges from $160(R=640)$ to $800(R=40)$. As expected, all curves collapse onto a single curve, which, in turn, supports that the BAAW with finite $R$ should belong to the DI class, irrespective of how large $R$ is.

\section{SUMMARY AND DISCUSSION}

To summarize, we studied the critical behavior of the BAAW with four offspring. We first estimated the critical exponents for the case $R=\infty$, which was first reported in Ref. [12]. Our estimates of the critical point and the critical exponents are more accurate than Ref. 12]. Our finding reconfirms that the $\mathrm{BAAW}_{\infty}$ does not belong to the directed Ising (DI) class.

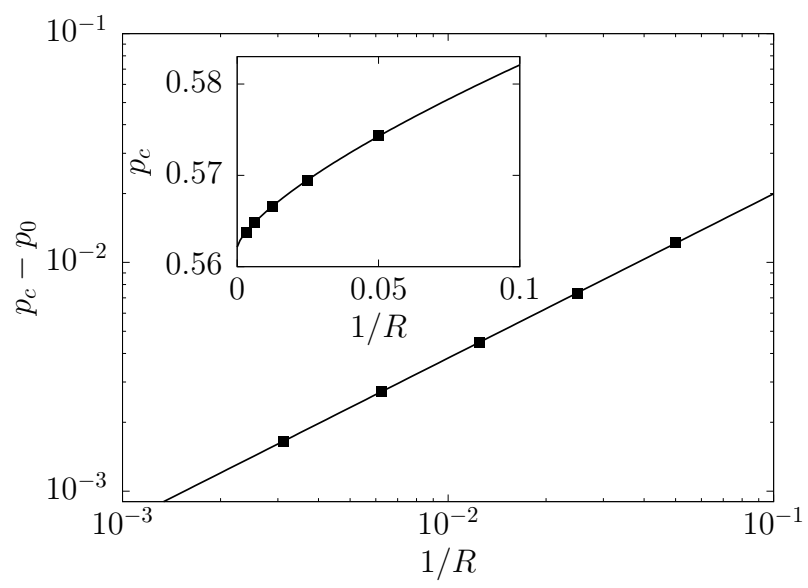

FIG. 7. Double logarithmic plot of $p_{c}-p_{0}$ vs. $1 / R$. A powerlaw fitting shows that $1 / \phi \approx 0.72$, which is also depicted as a straight line. Inset: Plot of $p_{c}$ vs. $1 / R$. The curve shows the fitting function.

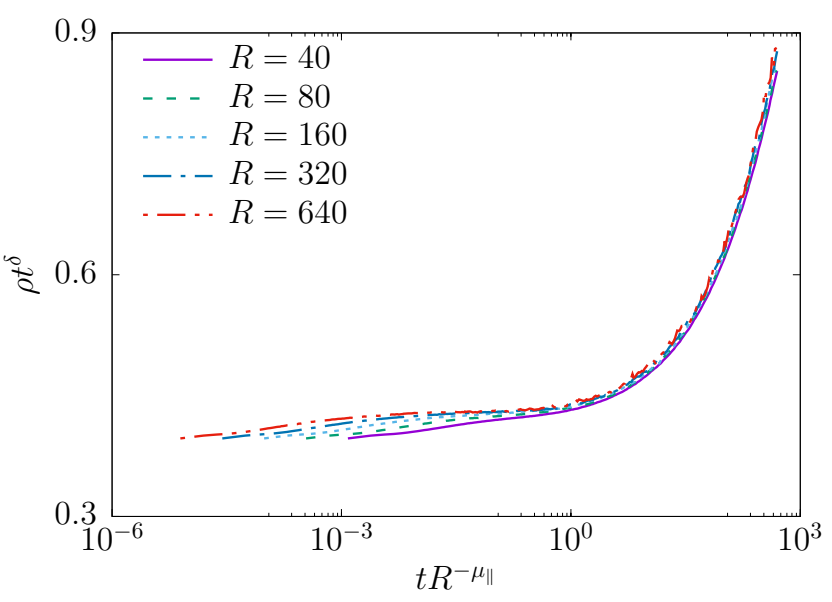

FIG. 8. Semilogarithmic plot of $\rho t^{\delta}$ vs. $t R^{-\mu_{\|}}$with $\mu_{\|}=1.82$ for $R=40,80,160,320$, and 640 , right to left. Asymptotic behavior is well collapsed into a single curve.

To elucidate the origin of the different critical behavior, we studied the crossover, occurring for finite $R$. We found that the BAAW with finite $R$ does belong to the DI universality class. We, furthermore, estimated the crossover exponent $\phi=1.39(4)$, by studying the behavior of the critical point for large $R$. Since only the case of $R=\infty$ is different from the DI class, we conclude that the different critical behavior of the $\mathrm{BAAW}_{\infty}$ is due to the long-range attraction.

In contrast, the bias in the $\mathrm{BAAW}_{\infty}$ was argued to be short-range interaction in Ref. [12]. This argument is based on the fact that the BAAW with odd number of offspring is found to belong to the directed percolation universality class irrespective of whether $R=0$ or $R=$ $\infty$, which is clearly different from the long-range jump dynamics [11, 15, 16].

However, the loss of long-range property of the bias for the case with odd $m$ in fact originates from its dynamics. To clarify this point, let us consider a situation, where there are only two particles in the (infinite) system and these particles are separated by $r$ which is assumed very large. For the bias (or, equivalently, the long-range attraction) to change the critical behavior, it is necessary that the long-time dynamics of this system should depend on $r$ as $r \rightarrow \infty$. Otherwise, the bias is just a local interaction.

If the number of offspring is odd $(m=2 \ell-1)$, then each particle can undergo a (effectively) spontaneous annihilation by the combination of branching and pairannihilation dynamics $(A \rightarrow 2 \ell A \rightarrow 0)$ before these two particles, more precisely two clusters of particles, merge. Thus, the survival probability that two clusters exist up to time $t$ does hardly depend on $r$ when $r \gg t$. At the critical point, this survival probability should decay as $t^{-2 \delta^{\prime}}$ for $1 \ll t(\ll r)$. In other words, the fate of each initial particle (more accurately the fate of each cluster) does not depend on the presence of other cluster and the 
long-time dynamics does not depend on $r$ for large $r$. Hence the bias becomes a (effectively) local interaction.

On the other hand, if the number of offspring is even as we have studied in this paper, then the system cannot fall into the absorbing state until initial two particles meet, even if the system is in the absorbing phase. Hence, the characteristic time $\tau$ depends on $r$ and diverge as $r \rightarrow \infty$. Since $\tau \sim r$ (biased diffusion) for infinite range of attraction and $\tau \sim r^{2}$ (normal diffusion) for finite range of attraction as $r \rightarrow \infty$ (in the absorbing phase), the longtime behavior differs only when the range of attraction is infinite. This again explains why $\mathrm{BAAW}_{\infty}$ is special.

Another direction of generalization of the $\mathrm{BAAW}_{\infty}$ is to associate the strength of the attraction with the distance to the nearest particle. One possibility is to modify the probability that a particle hops toward the nearest particle by $\frac{1}{2}+\epsilon r^{-\sigma}$, where $r$ is the distance to the nearest particle and $\sigma$ is a fixed number. Clearly, the $\mathrm{BAAW}_{\infty}$ corresponds to the case of $\sigma=0$. Our preliminary sim- ulation shows that the critical exponents of the BAAW with even $m$ indeed does depend on $\sigma$, as typical models with long-range interaction [11, 15, 16]. This again shows that the different universal behavior observed in Ref. [12] is due to the long-range interaction. Detailed analysis will be published elsewhere [23].

\section{ACKNOWLEDGMENTS}

This work was supported by the Basic Science Research Program through the National Research Foundation of Korea (NRF) funded by the Ministry of Science and ICT (Grant No. 2017R1D1A1B03034878) and by the Catholic University of Korea, research fund 2019. The author furthermore thanks the Regional Computing Center of the University of Cologne (RRZK) for providing computing time on the DFG-funded High Performance Computing (HPC) system CHEOPS.
[1] P. Grassberger, F. Krause, and T. von der Twer, A new type of kinetic critical phenomenon, J. Phys. A 17, L105 (1984).

[2] H. Takayasu and A. Y. Tretyakov, Extinction, survival, and dynamical phase transition of branching annihilating random walk, Phys. Rev. Lett. 68, 3060 (1992).

[3] M. H. Kim and H. Park, Critical behavior of an interacting monomer-dimer model, Phys. Rev. Lett. 73, 2579 (1994)

[4] N. Menyhárd, One-dimensional non-equilibrium kinetic ising models with branching annihilating random walk, J. Phys. A 27, 6139 (1994).

[5] K. E. Bassler and D. A. Browne, Nonequilibrium critical dynamics of a three species monomer-monomer model, Phys. Rev. Lett. 77, 4094 (1996).

[6] H. Hinrichsen, Stochastic lattice models with several absorbing states, Phys. Rev. E 55, 219 (1997).

[7] J. Kockelkoren and H. Chaté, Absorbing phase transitions of branching-annihilating random walks, Phys. Rev. Lett. 90, 125701 (2003).

[8] W. Hwang, S. Kwon, H. Park, and H. Park, Critical phenomena of nonequilibrium dynamical systems with two absorbing states, Phys. Rev. E 57, 6438 (1998).

[9] G. Ódor and N. Menyhard, Crossovers from parity conserving to directed percolation universality, Phys. Rev. E 78, 041112 (2008).

[10] S.-C. Park and H. Park, Three different routes from the directed ising to the directed percolation class, Phys. Rev. E 78, 041128 (2008).

[11] D. Vernon and M. Howard, Branching and annihilating Lévy flights, Phys. Rev. E 63, 041116 (2001)

[12] B. Daga and P. Ray, Universality classes of absorbing phase transitions in generic branching- annihilating particle systems with nearest-neighbor bias, Phys. Rev. E 99, 032104 (2019)

[13] H.-K. Janssen, On the nonequilibrium phase transition in reaction-diffusion systems with an absorbing stationary state, Z. Phys. B 42, 151 (1981).

[14] P. Grassberger, On phase transitions in Schlögl's second model, Z. Phys. B 47, 365 (1982).

[15] H. K. Janssen, K. Oerding, F. van Wijland, and H. J. Hilhorst, Lévy-flight spreading of epidemic processes leading to percolating clusters, Eur. Phys. J. B 7, 137 (1999).

[16] H. Hinrichsen and M. Howard, A model for anomalous directed percolation, Eur. Phys. J. B 7, 635 (1999).

[17] S.-C. Park, High-precision estimate of the critical exponents for the directed ising universality class, J. Kor. Phys. Soc. 62, 469 (2013).

[18] S.-C. Park, Critical decay exponent of the pair contact process with diffusion, Phys. Rev. E 90, 052115 (2014).

[19] H. Hinrichsen, Non-equilibrium critical phenomena and phase transitions into absorbing states, Adv. Phys. 49, 815 (2000).

[20] S.-C. Park, Order-parameter critical exponent of absorbing phase transitions in one-dimensional systems with two symmetric absorbing states, arXiv:2002.03143.

[21] I. D. Lawrie and S. Sarbach, in Phase Transitions and Critical Phenomena, Vol. 9, edited by C. Domb and J. L. Lebowitz (Academic Press, London, 1984).

[22] S.-C. Park and H. Park, Crossover from the pair contact process with diffusion to directed percolation, Phys. Rev. E 73, 025105(R) (2006).

[23] S.-C. Park, Branching annihilating random walks with long-range attraction in one dimension, arXiv:2003.02434 